\title{
SPATIALLY RESOLVED OBSERVATIONS OF THE BIPOLAR OPTICAL OUTFLOW FROM THE BROWN DWARF 2MASS J12073347-3932540*
}

\author{
E. T. Whelan ${ }^{1,2}$, T. P. RAY ${ }^{1}$, F. Comeron ${ }^{3}$, F. Bacciotti ${ }^{4}$, ANd P. J. Kavanagh ${ }^{5}$ \\ ${ }^{1}$ Dublin Institute for Advanced Studies, School of Cosmic Physics, 31 Fitzwilliam Place, Dublin 2, Ireland \\ ${ }^{2}$ UJF-Grenoble 1/CNRS-INSU, Institut de Planétologie et d'Astrophysique de Grenoble (IPAG) UMR 5274, Grenoble F-38041, France \\ ${ }^{3}$ ESO, Karl-Schwarzschild-Strasse 2, D-85748 Garching, Germany \\ ${ }^{4}$ INAF-Osservatorio Astrofisico di Arcetri, Largo E. Fermi 5, I-50125 Firenze, Italy \\ ${ }^{5}$ Institut für Astronomie und Astrophysik, Kepler Center for Astro and Particle Physics, Eberhard Karls Universität, D-72076 Tübingen, Germany \\ Received 2012 August 31; accepted 2012 October 25; published 2012 December 3
}

\begin{abstract}
Studies of brown dwarf (BD) outflows provide information pertinent to questions on BD formation, as well as allowing outflow mechanisms to be investigated at the lowest masses. Here new observations of the bipolar outflow from the $24 M_{\mathrm{JUP}}$ BD 2MASS J12073347-3932540 are presented. The outflow was originally identified through the spectro-astrometric analysis of the [O I] 36300 emission line. Follow-up observations consisting of spectra and [S $\mathrm{SI}], R$-band and $I$-band images were obtained. The new spectra confirm the original results and are used to constrain the outflow position angle (P.A.) at $\sim 65^{\circ}$. The $[\mathrm{O}$ I $] \lambda 6300$ emission line region is spatially resolved and the outflow is detected in the [S II] images. The detection is firstly in the form of an elongation of the point-spread function (PSF) along the direction of the outflow P.A. Four faint knot-like features (labeled A-D) are also observed to the southwest of 2MASS J12073347-3932540 along the same P.A. suggested by the spectra and the elongation in the PSF. Interestingly, D, the feature furthest from the source, is bow shaped with the apex pointing away from 2MASS J12073347-3932540. A color-color analysis allows us to conclude that at least feature D is part of the outflow under investigation while A is likely a star or galaxy. Follow-up observations are needed to confirm the origin of $\mathrm{B}$ and $\mathrm{C}$. This is a first for a BD, as BD optical outflows have to date only been detected using spectro-astrometry. This result also demonstrates for the first time that BD outflows can be collimated and episodic.
\end{abstract}

Key words: ISM: jets and outflows - stars: pre-main sequence

Online-only material: color figures

\section{INTRODUCTION}

The brown dwarf (BD) 2MASS J12073347-3932540 (hereafter 2M1207A), with an estimated mass of $24 M_{\mathrm{JUP}}$ (Mohanty et al. 2007), has garnered much attention in recent years. Not only does it exhibit young star-like properties, i.e., it is a strong accretor with a detected accretion disk (Riaz et al. 2012a, 2012b; Riaz \& Gizis 2008; Mohanty et al. 2003, 2005) and bipolar outflow (Whelan et al. 2007), but it also has a planetary mass companion 2M1207B. 2M1207B was first detected by Chauvin et al. (2004) using direct imaging and it is one of only a small number of exoplanets to have been detected in this way. The 2M1207 system is a member of the TW Hya association (TWA). TWA is one of the closest young stellar associations to the Sun with an estimated distance of 47-67 pc and an age of $\sim 10 \mathrm{Myr}$ (Barrado y Navascués 2006). Thus TWA is a key reference for the study of star, BD and planet formation, and associated phenomena. It is the interesting properties of 2M1207 combined with its mass at the lower end of the BD mass spectrum and relatively close proximity that have made this object the target of studies aimed at understanding BD and planet formation.

In this paper, new observations of the bipolar outflow driven by $2 \mathrm{M} 1207 \mathrm{~A}$ are presented. As studies of outflow properties can provide information on the central engine of the driving source, observations of $\mathrm{BD}$ outflows can contribute much to

\footnotetext{
* Based on data collected by UVES (281.C-5025(A)) and FORS1 (380.C-0412(A)) observations at the VLT on Cerro Paranal (Chile) which is operated by the European Southern Observatory (ESO). Data collected from the ESO archive (071.C-0432(A), 085.C0238(A), 078.C-0158(A, B)) are also used in the analysis presented here.
}

the debate on the origin and evolution of BDs. For example, the kinematics and position angle (P.A.) of the outflow can be used to infer the disk inclination and P.A. (Whelan et al. 2009b). Episodic or knotty outflows point to variable accretion activity, and precession or wiggling in an outflow can be interpreted as evidence of an unresolved companion (Whelan et al. 2010). In addition, measurements of the mass outflow to accretion rate $\left(\dot{M}_{\text {out }} / \dot{M}_{\text {acc }}\right)$ are relevant to jet launching models which in turn affect models of disk evolution and thus planet formation (Ferreira et al. 2006; Hartigan et al. 1995). One possible difference between BDs and low-mass stars is that first results indicate that $\dot{M}_{\text {out }} / \dot{M}_{\text {acc }}$ may be higher for substellar objects than low-mass stars. For low-mass stars, it is currently estimated at $\sim 10 \%$; however, in BDs this ratio has been found to be significantly higher and, in the most extreme cases, the two rates are found to be comparable (Bacciotti et al. 2011; Whelan et al. 2009b). This is significant as it may help to explain why BDs never accrete enough matter to become stars (Machida et al. 2009). Better observations of a larger number of BDs are needed to confirm these results, to properly understand $\mathrm{BD}$ accretion/outflow properties and thus to compare BD properties with low-mass stars.

BD outflows have mainly been studied through spectroscopy and especially the spectro-astrometric analysis of their forbidden emission line (FEL) regions. The first indications that BDs could drive outflows came from the detection of FELs in their optical spectra (Fernández \& Comerón 2001). Spectro-astrometry (SA), which involves using Gaussian fitting to measure the centroid of an emission region as a function of wavelength/velocity, was used to confirm that these FEL regions were extended and originated in an outflow (Whelan et al. 2005). Since the first 


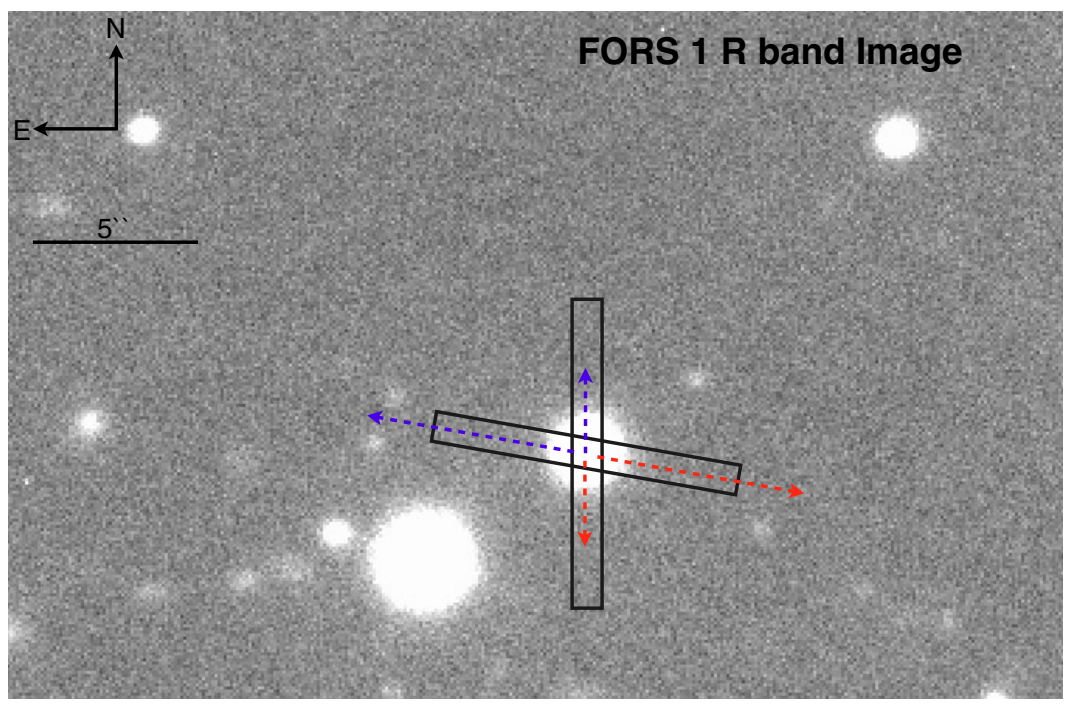

$\mathrm{PA}=0^{\circ}, 2005-16-05$

$\mathrm{PA}=80^{\circ}, 2008-17-07$

Figure 1. FORS1 $R$-band image of 2M1207 with the UVES slits and slit P.A.s shown. The slits are drawn to scale. The spectro-astrometric offsets measured at the two slit P.A.s, for the blueshifted and redshifted [O I] 26300 emission, are represented by the blue and red arrows. The sizes of the arrows do not represent the magnitude of the measured offsets. However, they accurately represent the relative magnitudes between the two observations, in that the offsets measured at P.A. $=80^{\circ}$ are approximately twice those measured at P.A. $=0^{\circ}$.

(A color version of this figure is available in the online journal.)

detection, four further young BD candidates have been found to drive outflows using this method. Imaging studies of BD outflows have rarely been conducted and the mapping of the molecular outflow from the BD ISO-Oph 102 with the Submillimeter Array is the highest quality image in existence at present. The very low mass star (VLMS) Par-Lup3-4 with a mass just above the substellar limit (Comerón et al. 2003) has been studied with a combination of imaging and spectroscopy, and a bipolar outflow was detected using both methods (Comerón \& Fernández 2011; Fernández \& Comerón 2005). Previous to this work it was the lowest mass protostar where an optical outflow had even been marginally detected using imaging. For Par-Lup3-4, the $[\mathrm{S} \mathrm{II}]$ image is well extended along the outflow P.A. and a Herbig Haro (HH) object (HH 600) is also detected. Comerón \& Fernández (2011) estimate a proper motion of $168 \pm 30 \mathrm{~km} \mathrm{~s}^{-1}$ for HH 600. Wang \& Henning (2006) carried out a survey for $\mathrm{HH}$ objects in the Chamaeleon I molecular cloud with the aim of searching for optical outflows from BDs. While $18 \mathrm{HH}$ objects and $\sim 40 \mathrm{BD}$ candidates were identified, they found no persuasive evidence that any of the BDs were related to the $\mathrm{HH}$ objects.

Optical spectra of 2M1207A obtained in 2006 May contained double-peaked [O I] 26300 emission which was tracing a bipolar outflow (Whelan et al. 2007). This was an exciting discovery as it confirmed 2M1207A as the lowest mass galactic object known to drive an outflow. The observations presented here can be divided into three separate results. First, new optical spectra confirm the original detection and a comparison with the 2006 spectra allows an estimate of the outflow P.A. to be made. Second, we present an [S II] image in which the outflow is detected close to the source in the form of an elongation in the point-spread function (PSF). Third, a series of knot-like features are seen in the $[\mathrm{S}$ II] image along the P.A. suggested by the spectro-astrometric study. We explore the possibility that these features are shocks in the 2M1207A outflow. Below these new observations are described, their significance discussed, and the best direction for future studies of BD outflow activity considered.

\section{OBSERVATIONS AND DATA ANALYSIS}

Both spectroscopic and imaging studies of the outflow driven by 2M1207A have been conducted with the European Southern Observatory's (ESO) Very Large Telescope (VLT), using the UV-Visual Echelle Spectrometer (UVES) and the FOcal Reducer/low-dispersion Spectrograph 1 (FORS1; Dekker et al. 2000; Appenzeller et al. 2000; Szeifert et al. 1998). Follow-up observations to the original UVES data were made with FORS1 in 2008 January/March and UVES in 2008 August. Starting first with the UVES 2008 spectroscopy, observations were carried out with the CD3 grating and a central wavelength of $6000 \AA$, providing spectra with a wavelength range of 4900-7100 and spectral resolution $R=40,000$. The slit width and pixel scale were $1^{\prime \prime}$ and 0 '. 182 , respectively, and the seeing was $\sim 0$.' 8 . Two observations with exposure time totaling $2900 \mathrm{~s}$ were taken. The UVES 2006 data had the same instrumental setup except that the central wavelength was set at $5800 \AA$, meaning that the wavelength range included the $\mathrm{H} \beta$ line at $4861 \AA$. Indeed, $2 \mathrm{M} 1207 \mathrm{~A}$ has been observed numerous times with UVES and the spectra are available in the ESO data archive. However, observations were generally aimed at studying accretion and were not optimized for outflow studies. This means that the exposure time was too low to detect the forbidden emission.

The UVES 2006 observations, published in Whelan et al. (2007), were made with the slit aligned with a P.A. of $0^{\circ}$, i.e., north-south. For the 2008 spectra, the P.A. was set at the parallactic angle which varied between $75^{\circ}$ and $85^{\circ}$ over the course of the observation. By comparing the analysis of the 2008 spectra with the 2006 spectra, the P.A. of the outflow can be probed and, for the purpose of this, we take the slit P.A. to be $80^{\circ}$. Figure 1 presents the different slit P.A.s used and the direction on the sky to which the offsets correspond. 

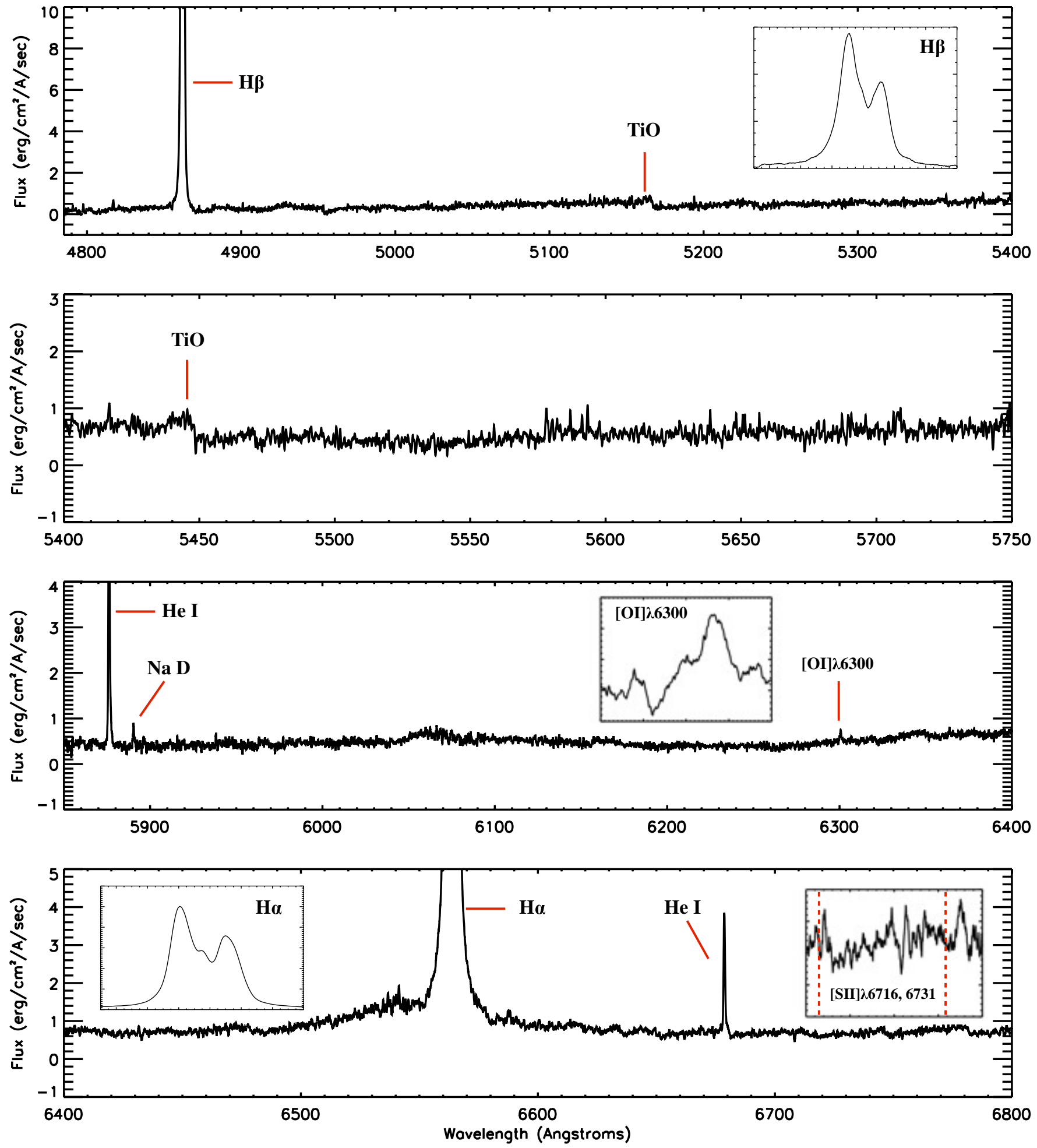

Figure 2. Full spectral range of the UVES observations aimed at studying the 2M1207A outflow. This one-dimensional spectrum was extracted from the source position using the ESO UVES pipeline and was taken on 2006 May 16. $\mathrm{H} \alpha$ and $\mathrm{H} \beta$ are by far the strongest lines and while [O I] 66300 line is detected no [S II] emission is detected above the noise at the source position. In the inset showing the region of the [S II] $] \lambda 26716,6731$ lines, the dashed red lines mark the $0 \mathrm{~km} \mathrm{~s}^{-1}$ position for the two emission regions.

(A color version of this figure is available in the online journal.)

The spectra were reduced using standard routines for bias subtraction, flat fielding, and wavelength calibration, provided by the Image Reduction and Analysis Facility (IRAF). In order to analyze the spectra using SA, the two-dimensional spectra corresponding to each individual order had to be extracted and wavelength calibrated using the Identify, Reidentify, Fitcoords, and Transform routines. Continuum subtraction was carried out using the Continuum routine. Overall, the process followed for the SA was the same as outlined in Whelan et al. (2007) including the smoothing of the data using Gauss 
(a)

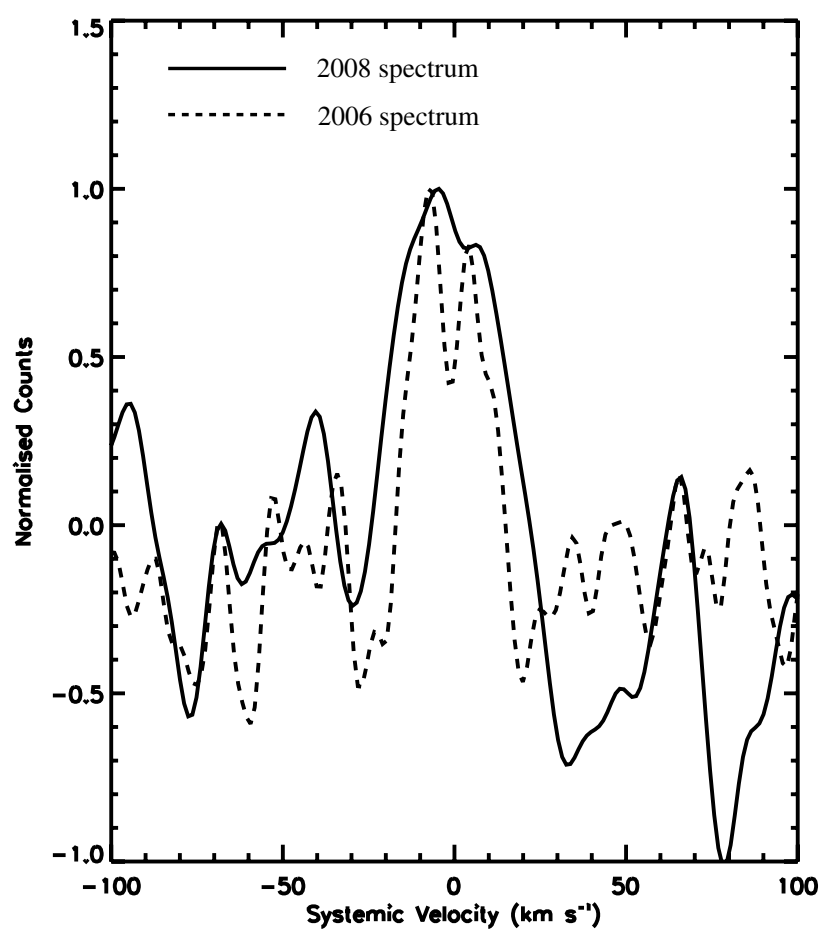

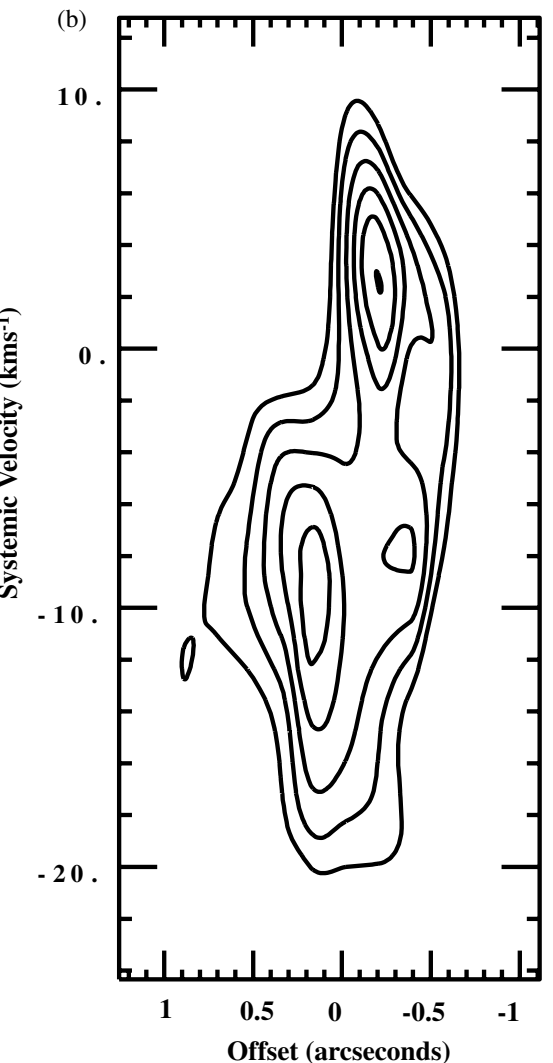

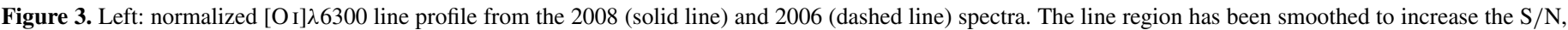

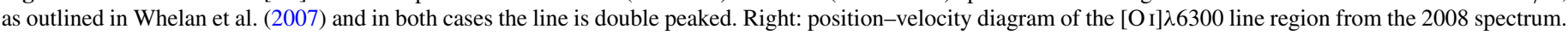

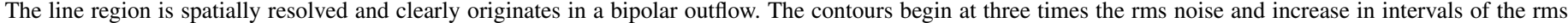

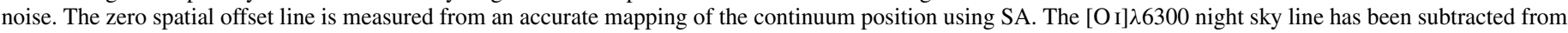
the spectrum.

to increase the signal-to-noise ratio $(\mathrm{S} / \mathrm{N})$. Also see Whelan \& Garcia (2008) for further details on the spectro-astrometric technique.

Imaging with FORS1 was carried out using the $[\mathrm{S} \mathrm{II}]+62$ narrowband filter and the $R$-BESS and $I$-BESS broadband filters in 2008 March. All observations were made with the highresolution collimator and a binning of $2 \times 2$, giving a pixel scale of 0 '.125. Again standard IRAF routines were used for bias subtraction and flat fielding. For the [S II] images a total of $2 \times 30$ minute exposures were made. The exposures were taken consecutively, however the seeing deteriorated from $\sim 0$.' 6 to 0.9 between the two exposures. The total exposure time for the broadband images was 15 minutes. Although the [O I] 26300 line had previously been detected in the 2M1207A spectrum and shown to trace an outflow, for the purposes of imaging, [S II] was preferred over [O I] due to the relatively bright background emission in [OI] from the atmosphere. To help with the analysis of the [S II] FORS1 images, SUSI2 I-band images were obtained from the ESO archive and are further discussed in the Section 3.

As dithering was not used to obtain the I-band images, fringing effects which could not be removed using the flat fields presented a problem to the analysis of faint emission in the vicinity of 2M1207A. To remove the fringing, separate $I$-band observations were identified and downloaded from the ESO archive. The closest ones in time were taken on 2008 February 11, which is about one month before our observations of 2M1207, and had been originally obtained to image SN
$2007 Y$ in the galaxy NGC 1187 . The galaxy has a relatively large angular size that fills a significant fraction of the field of view (FOV) of FORS1. However, the area of the detector where 2M1207 was later imaged corresponded to a region in the distant outskirts of the galaxy with no obvious structure. Thanks to this, it was possible to construct a fringe pattern frame suitable for the region immediately surrounding 2M1207 by combining and median-filtering the eight available exposures of NGC 1187 , uncorrected for telescope offsets between the exposures. The fringe pattern obtained was visually confirmed to be very similar in appearance to that in the 2M1207 exposures. Next, an average sky background value near the position of 2M1207 in the detector was subtracted from both the 2M1207 and fringing pattern images. The background-subtracted fringing pattern image was then scaled by a numerical factor and subtracted from the background-subtracted image of 2M1207. Based on visual inspection of the resulting image, this last part of the process was iterated with different scaling factors applied to the background-subtracted fringing image, minimizing the residual fringing pattern in the image of $2 \mathrm{M} 1207$. As dithering was used to obtain the SUSI2 images of 2M1207 identified in the ESO archive, construction of a fringing pattern from separate images was not needed in this case. Instead, the frames containing the target were combined with median filtering to construct a sky frame, including fringing, to be subtracted from the individual images. The sky background remained very stable during all the SUSI2 observations, thus leaving no noticeable fringing residuals in the sky-subtracted images. 


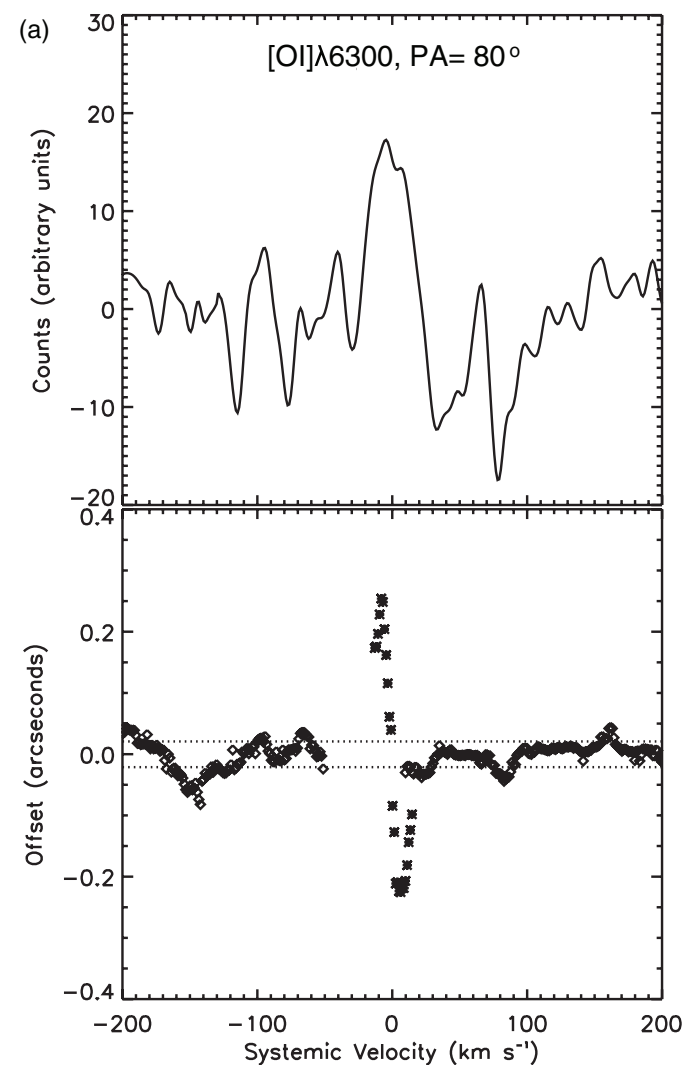

(b)

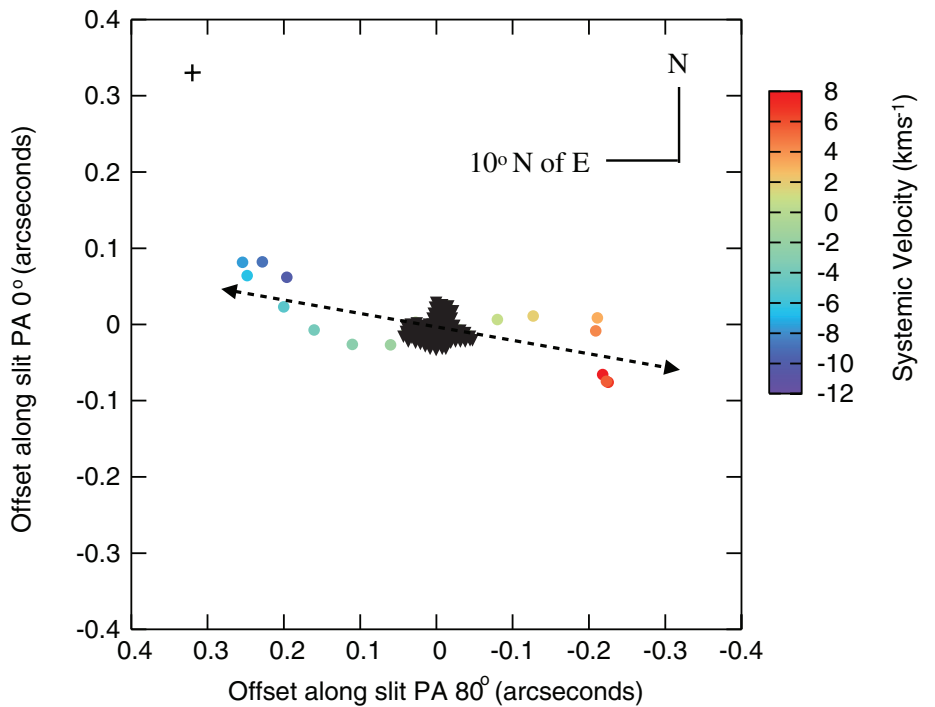

Figure 4. Left: SA of the 2008 [O I] 76300 line. The SA clearly confirms what is revealed in the position-velocity diagram in Figure 3, that the blue and red peaks are offset in opposing directions. The dashed line marks the $1 \sigma$ error in the centroid fitting. Right: the spectro-astrometric signatures measured in the [O I] 26300 line in 2006 and 2008 are combined to constrain the outflow P.A. Note that the $x$ - and $y$-axes do not represent orthogonal slit P.A.s but rather P.A.s of $0^{\circ}$ and $80^{\circ}$.

(A color version of this figure is available in the online journal.)

\section{RESULTS}

\subsection{Spectroscopy and Spectro-astrometry}

In Figure 2 the one-dimensional extracted spectrum from the UVES 2006 data is shown. This spectrum was extracted using the ESO UVES pipeline and the purpose here is to show the full extent of the UVES spectra and to mark the strongest lines. The 2006 UVES spectrum is shown as the 2008 spectrum does not include the region of the $\mathrm{H} \beta$ line. In this study, SA is applied to the $[\mathrm{O} \mathrm{I}] \lambda 6300, \mathrm{H} \alpha$, and $\mathrm{H} \beta$ lines to search for evidence of the outflow, and a comparison is made between the 2006 and 2008 spectra. Beginning with the [O I] $\lambda 6300$ line, Figure 3(a) compares the line profiles of the $[\mathrm{O} I] \lambda 6300$ emission in the 2006 data and in the 2008 data. In both cases, the line is double peaked with the blueshifted and redshifted peaks at $\sim-8 \mathrm{~km} \mathrm{~s}^{-1}$ and $+4 \mathrm{~km} \mathrm{~s}^{-1}$, respectively. The small radial velocities of the peaks agree with the hypothesis that 2M1207A has a near-edgeon accretion disk and, therefore, an outflow close to the plane of the sky. The inclination of the 2M1207A disk has recently been estimated by Riaz et al. (2012a, 2012b) at $57^{\circ}-69^{\circ}$. The kinematics of the outflow and spectro-astrometric results would place the inclination at the upper end of this scale. In the 2006 spectra, the emission peaks were found to be offset in opposite directions by $\sim 80$ mas confirming the origin of the emission in an outflow. In Figure 3(b) the position-velocity diagram of the 2008 [O I] 26300 emission region is shown. The two peaks are clearly seen and, interestingly, they are spatially offset in opposite directions, verifying the conclusion of Whelan et al. (2007) and revealing that the P.A. of the 2M1207A outflow lies closer to a P.A. of $90^{\circ}$ than $0^{\circ}$. This is the first observation where a BD FEL region is spatially resolved and therefore where SA is not necessary to demonstrate origin in an outflow. In Figure 4 the P.A. of the outflow is recovered by plotting the offsets at $0^{\circ}$ against the offsets at $80^{\circ}$ as a function of systemic velocity. Taking into account the fact that the two slit P.A.s were not perpendicular, the outflow P.A. is constrained at $\sim 65^{\circ} \pm 10^{\circ}$. Assuming that the outflow is perpendicular to the disk, this gives a likely disk P.A. of $\sim 155^{\circ}$. The P.A. of the companion is $125.6 \pm 0.7$ (Mohanty et al. 2007). The difference of $\sim 30^{\circ}$ between the disk P.A. (inferred from the outflow P.A.) and the P.A. of the companion could be accounted for if the companion and disk are not coplanar (Riaz et al. 2012a, 2012b). Alternatively, projection effects could explain the difference. The difference between the outflow axis projected on the sky and the P.A. of the planet will be $90^{\circ}$ only at the time of the greatest elongations. For most of the orbit, it will be near but not exactly $90^{\circ}$ for a close to, but not exactly, edge-on orbital plane, depending on the position on the orbit.

In addition to FELs, permitted emission lines are also strong tracers of outflow activity in BDs and low-mass stars (Whelan et al. 2004, 2009a). Indeed $\mathrm{H} \alpha$ and $\mathrm{H} \beta$ emission is known to trace outflows and collimated jets (Bacciotti et al. 2011). Typically it is found that both outflow and accretion process contribute to the permitted line regions with the wing emission often tracing the outflow component (Takami et al. 2001; Whelan et al. 2004; Podio et al. 2008). SA is also used here to search for any contribution to the $\mathrm{H} \alpha$ and $\mathrm{H} \beta$ lines from the 2M1207A outflow. Whelan et al. (2007) analyzed the $2006 \mathrm{H} \alpha$ emission region and no evidence of an outflow component was found. The $\mathrm{H} \alpha$ and $\mathrm{H} \beta$ lines of 2M1207A are, 

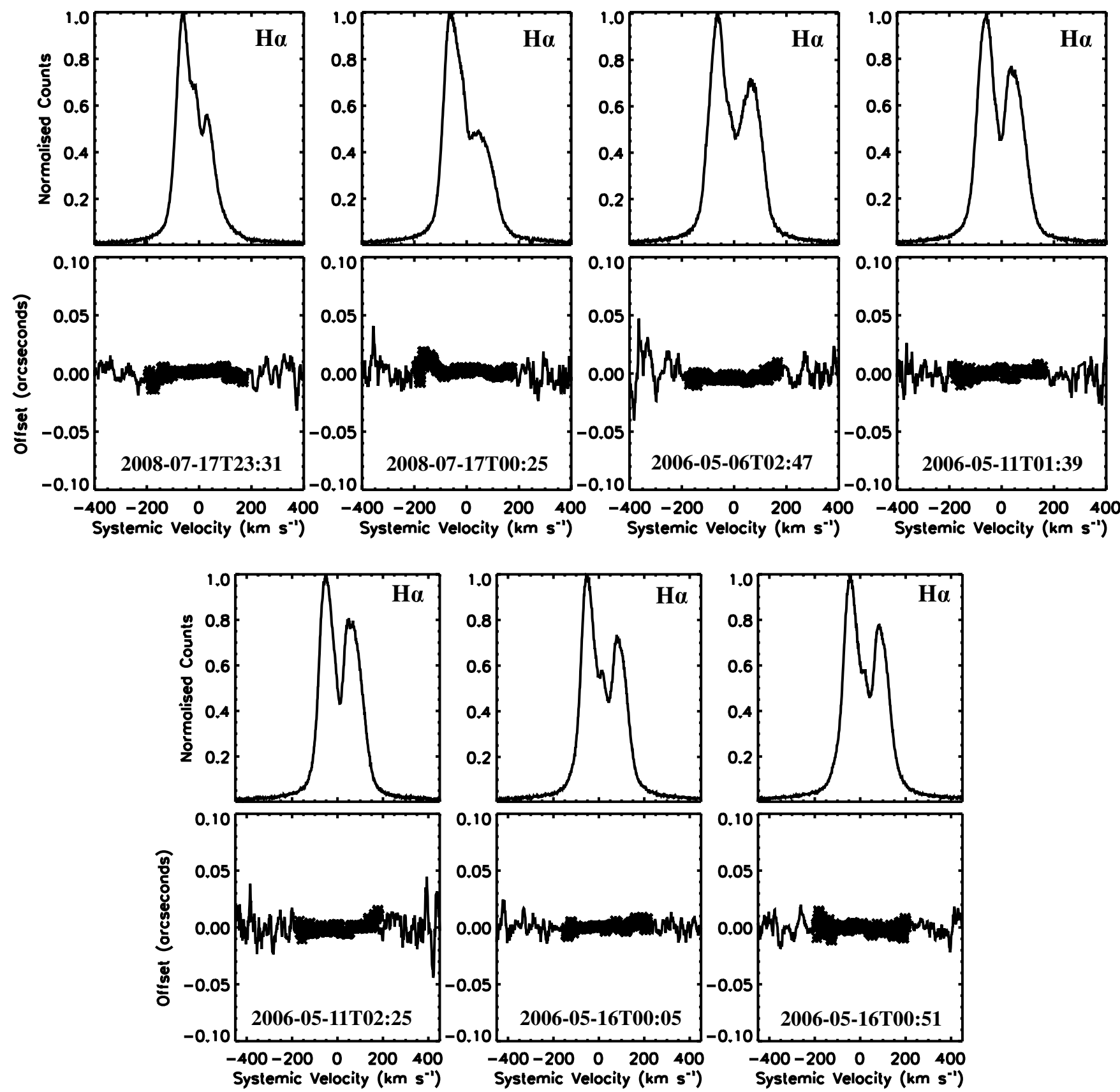

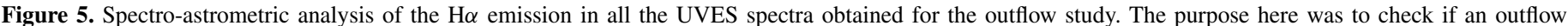

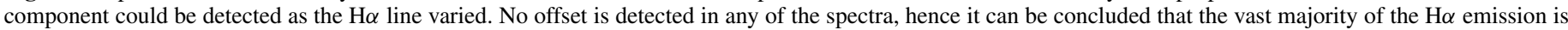

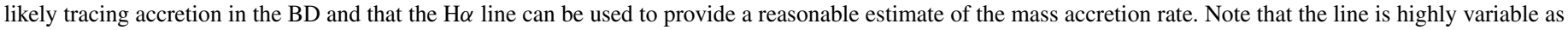
outlined by Scholz et al. (2005). The appearance of a small bump between the primary and secondary peaks was not previously detected in any variability study.

however, known to be highly variable (Scholz et al. 2005; Stelzer et al. 2007), and therefore one possibility that is important to consider is that the 2006 spectra were taken during a period of high accretion activity, making it very difficult to detect any outflow component. If spectra are taken during a period of lower accretion activity, the ratio between the emission from the outflow and the emission from the accretion could be more conducive to the detection of the outflow component. With this in mind, all the $\mathrm{H} \alpha$ emissions from all the spectra taken in 2006 and 2008 were analyzed using SA. For the analysis of the [O I] $\lambda 6300$ line, the spectra were median combined to increase the $\mathrm{S} / \mathrm{N}$ in the $\left[\mathrm{O}_{\mathrm{I}}\right] \lambda 6300$ line region. However, the $\mathrm{S} / \mathrm{N}$ in the region of the $\mathrm{H} \alpha$ line is high enough to analyze the spectra separately. Five individual spectra were obtained in 2006 with a further two in 2008. Figure 5 compares the $\mathrm{H} \alpha$ line profile shape and spectro-astrometric signature in all seven spectra. While the shape changes a lot (note the appearance of a small "bump" between the two main peaks), no spectro-astrometric signature is detected. Hence it can be argued that the majority of the emission is coming from close to the source, presumably from accretion, and that the $\mathrm{H} \alpha$ should provide a good estimate of the mass accretion rate of $2 \mathrm{M} 1207 \mathrm{~A}$. The small "bump" is interesting as it was not previously detected in the variability studies of Stelzer et al. (2007) or Scholz et al. (2005). Similarly we compare the 

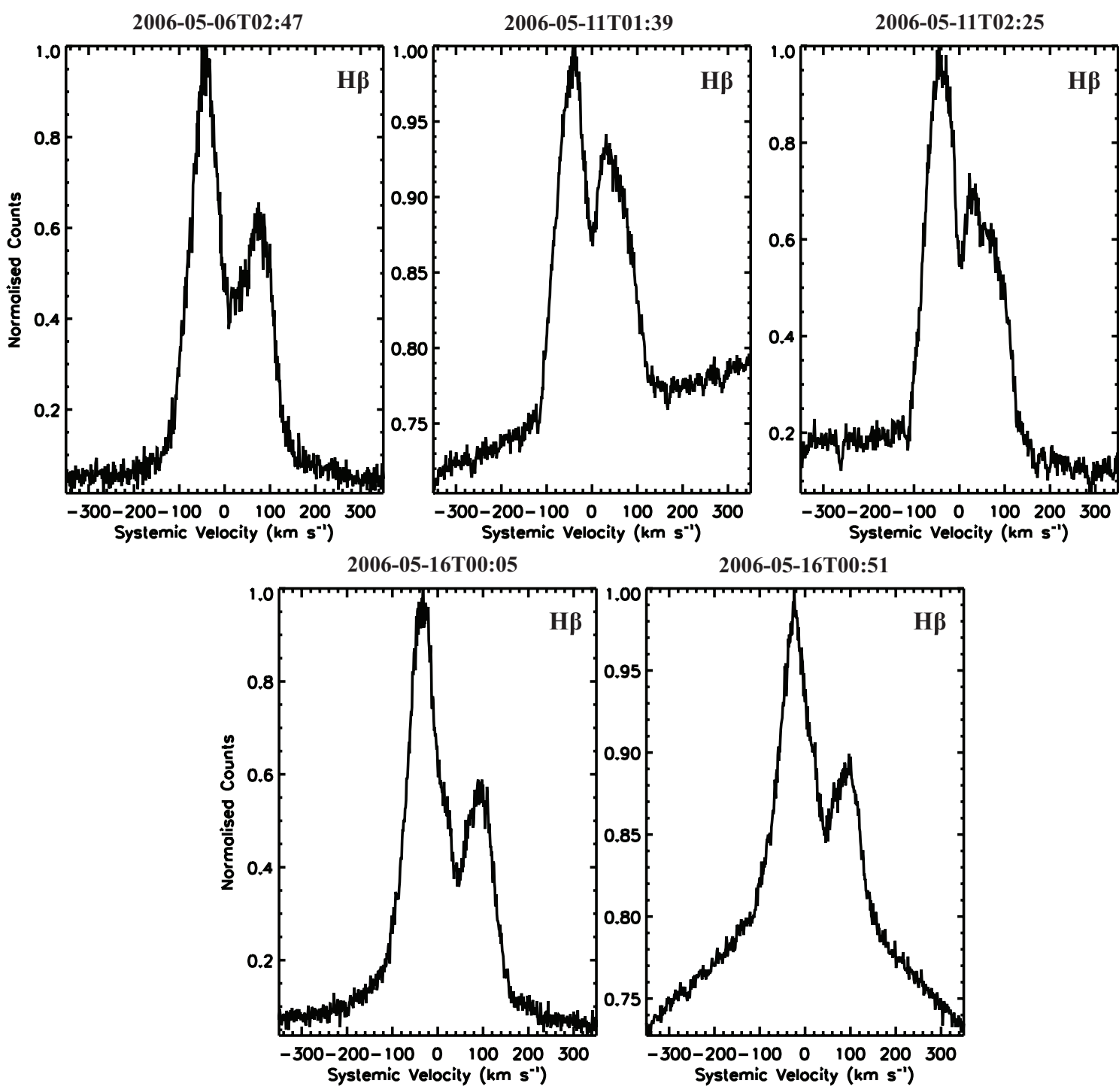

Figure 6. Comparing the $\mathrm{H} \beta$ line profiles in the 2006 spectra. Both the line and continuum vary. No spectro-astrometric signal was detected for the $\mathrm{H} \beta$ line.

line profile shape of the $\mathrm{H} \beta$ and spectra-astrometric signature of the $\mathrm{H} \beta$ line. Again no spectro-astrometric signal is detected and only the line profiles are presented in Figure 6.

\section{2. [S II] Imaging: Elongated PSF}

The FORS1 [S II] image with an inset of a zoom on 2M1207A is shown in Figure 7. First, we chose to look for evidence of emission from the outflow close to the BD by analyzing the image with the best seeing $\left(\sim 0^{\prime \prime} 6\right)$. Interestingly, the PSF appears to be extended along the outflow P.A. suggested by the spectro-astrometric results. In Figure 8, the PSF of 2M1207A in the $[\mathrm{S} \mathrm{II}]$ image is compared with the PSF of two nearby stars in [S II] and with 2M1207A in $R$. Each PSF was fitted with an ellipse and the results compared. The 2M1207A PSF is more elliptical than the others and is elongated along a P.A. given by the elliptical fit at $62^{\circ} \pm 6^{\circ}$. This is consistent with the estimate of $65^{\circ} \pm 10^{\circ}$ for the outflow P.A. This agreement, along with the fact that the extension is only seen in the [S II] PSF of 2M1207A, is strong evidence against an artifact being the cause of the extension. Hence, we argue that we are in fact detecting the 2M1207A outflow in the [S II] image. However, note that no $[\mathrm{S} \mathrm{II}]$ emission is detected at the source position in the UVES spectra (see Figure 2). Why [S II] outflow emission should be detected in the FORS1 image and not in the UVES spectra requires further investigation. Using one-dimensional flux-calibrated spectra from the UVES pipeline, the flux of the 2M1207A [OI] 6300 emission line is estimated at $1 \times$ $10^{-16} \mathrm{erg} \mathrm{cm}^{-2} \mathrm{~s}^{-1}$ and the continuum flux included in the range of the $[\mathrm{S} \mathrm{II}]$ narrowband filter at $37 \times 10^{-16} \mathrm{erg} \mathrm{cm}^{-2} \mathrm{~s}^{-1}$ $\left(0.6 \times 10^{-16} \mathrm{erg} \mathrm{cm}^{-2} \mathrm{~s}^{-1} \AA^{-1}\right)$. Using UVES spectra of other $\mathrm{BD} / \mathrm{VLMS}$ where both [O I] and [S II] emission is detected, we measured the average flux ratio of the $[\mathrm{S}$ II $] \lambda 6716 /[\mathrm{O}$ I $] \lambda 6300$ and $[\mathrm{S}$ II $] \lambda 6731 /[\mathrm{O} \mathrm{I}] \lambda 6300$ lines and use these values to make a realistic estimate of the ratio between the [S II] and [OI] emission in 2M1207A. If this same ratio holds for 2M1207A, then the [S II] lines would have strengths of 0.18 and $0.3 \times$ $10^{-16} \mathrm{erg} \mathrm{cm}^{-2} \mathrm{~s}^{-1}$, respectively. This puts the [S II] line at around the $1 \sigma$ to $2 \sigma$ detection level, explaining its non-detection above the continuum.

Due to the difference in critical density between the $[\mathrm{OI}] \lambda 6300$ and $[\mathrm{S} \mathrm{II}] \lambda \lambda 6716,6731$ lines, the [OI] emission is stronger than the $[\mathrm{S} \mathrm{II}]$ emission at the source position and the [S II] emission is normally more extended. The critical densities of [O I] $\lambda 6300$ and [S II] 66731 lines, at $T \sim 8000 \mathrm{~K}$, can be taken as $2 \times 10^{6} \mathrm{~cm}^{-3}$ and $1.3 \times 10^{4} \mathrm{~cm}^{-3}$, respectively (Hartigan 


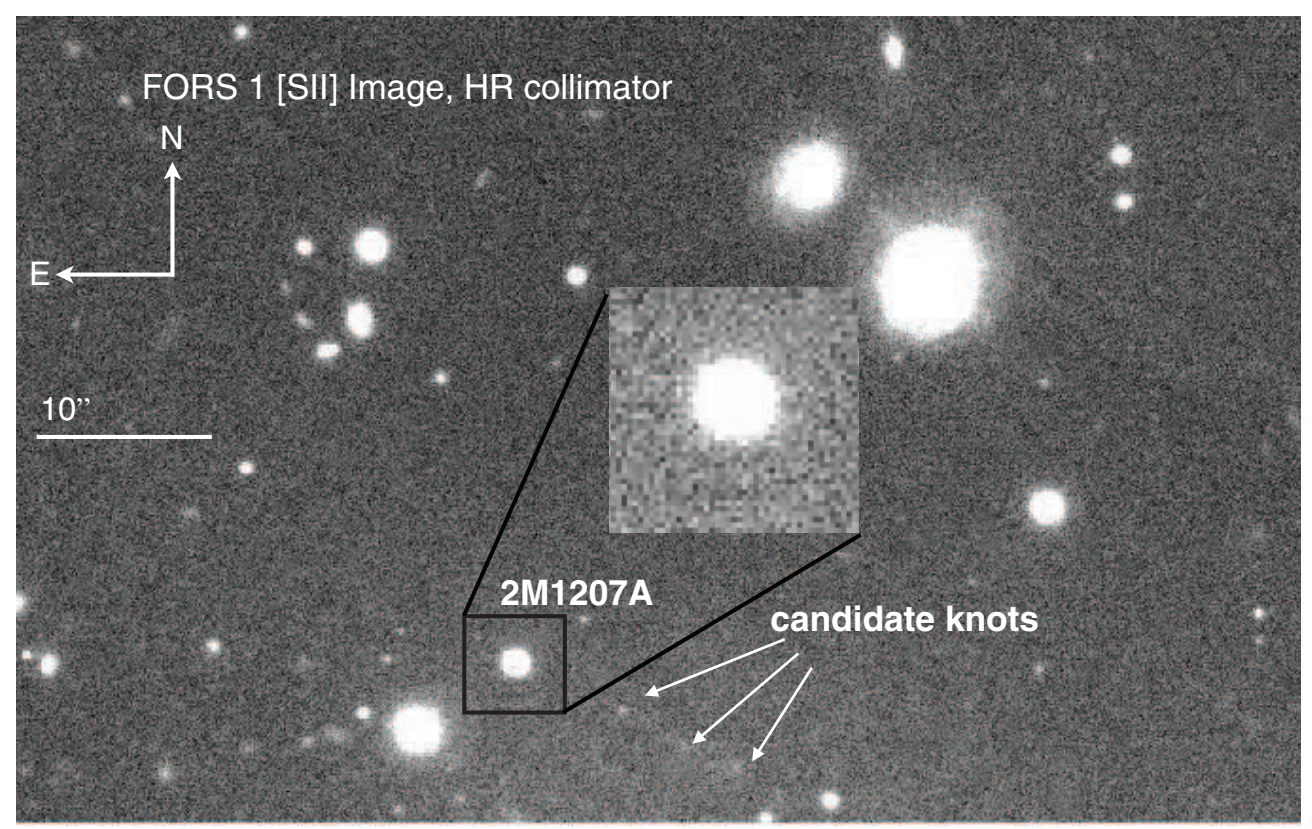

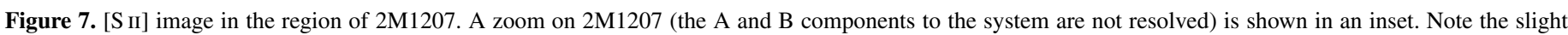

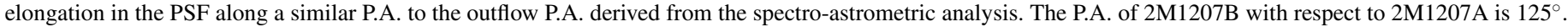

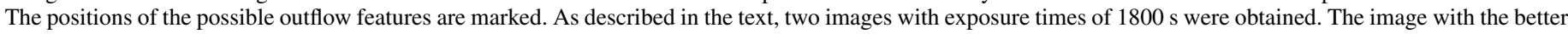
seeing is shown here.

et al. 1995). Thus, knots further out in a jet are brighter in [S II] than [O I] and this is illustrated, for example, for the Par-Lup3-4 outflow in Bacciotti et al. (2011). To check for faint extended $\left[\mathrm{S}\right.$ II] emission, we summed the spectra over $\pm 1^{\prime \prime}$ and the results are plotted in Figure 9. Indeed, once the spectra are summed in the spatial direction, both [S II] lines are detected at a velocity comparable to the $\left[\mathrm{O}_{\mathrm{I}}\right] \lambda 6300$ velocity. The spectra have been smoothed in the same way as the [O I] 6300 spectrum and also shown is the Li I photospheric line which is used to measure the systemic velocity. Spectra from 2006 and 2008 are compared and in both cases the [S II] $\lambda 6716$ line is brighter than the [S II] 6731 line indicating a low value of $n_{e}$ of $\sim 1000 \mathrm{~cm}^{-3}$ (Osterbrock 1988). The fact that some extended [S II] emission is detected in the UVES spectra supports our detection of the outflow in the image.

\section{3. [S II] Imaging: Extended Emission Features}

To the southwest of 2M1207A, a series of faint knot-like features which culminate in a bow-shock-shaped object at a distance of $\sim 16^{\prime \prime}$ are detected (see Figures 7 and 10). These will now be referred to as features $\mathrm{A}$ to $\mathrm{D}$, with $\mathrm{D}$ being the feature furthest from the BD. These sources seen in $[\mathrm{S}$ II $], R$, and $I$ are in the direction of the redshifted outflow and look very much "to the eye" like they could be outflow knots. They are well fitted with a P.A. of $245^{\circ}$ as expected for the redshifted flow. However, it is possible that A-D could be faint stars or galaxies and therefore further analysis is needed to confirm that they are $\mathrm{HH}$ objects forming part of the 2M1207A outflow. First, a [S II] $-I$-band subtraction was carried out to isolate any outflow emission. In Figure 10 the FORS 1 [S II], $R$-, and $I$-band images and an SUSI2 I-band image downloaded from the ESO archive are compared. The features are clearly detected in all bands. The $R$-BESS filter covers the range 5820-7320 $\AA$ and the $I$-BESS filter the range 6990-8370 А. Hence, both filters include outflow tracers, especially the $R$-BESS which covers strong tracers like $[\mathrm{O}$ I $] \lambda 6300, \mathrm{H} \alpha,[\mathrm{N}$ II $] \lambda 6583$, and [S II $] \lambda 6731$.
Outflow tracers such as [Fe II] $\lambda 7155$ and [Ca II] $\lambda 7291$ are found in the range of the $I$ band. However, as these lines are fainter than the lines in the range of the $R$ band and sometimes not present, the outflow emission in the $I$ band is generally significantly less than, for example, that of the $\left[\mathrm{S}_{\mathrm{II}}\right]$ narrowband filter (Brugel et al. 1981; Mundt et al. 1983). Therefore, I-band broadband images are routinely used to remove continuum emission from a narrowband image and thus to enhance any outflow emission. In Figure 11, a Gaussian-smoothed FORS1 [S II]- I image of the region where it was possible to remove the $I$-band fringing is shown. The emission regions $\mathrm{A}-\mathrm{D}$ are detected and the bow-shaped nature of feature $\mathrm{D}$ is obvious. The red line marks the position of outflow P.A. which well fits knot $\mathrm{D}$ in particular. The emission regions $\mathrm{B}$ and $\mathrm{C}$ are just above the noise in the [S II] $-I$ image while $A$ is the brightest.

To further eliminate the possibility that A-D are stars/ galaxies a [S II] $-I$ versus $R-I$ color-color analysis was carried out and is presented in Figure 12. An HH object is expected to display a blue broadband $R-I$ color due to the contribution of the $\mathrm{H} \alpha$ and [S II] $\lambda \lambda 6716,6731$ lines to the $R$ band, and an even bluer [S II] $-I$ color caused by the larger fraction of the [S II] filter passband in which [S II] emission is noticeable, in contrast with the redder $R-I$ and [S II] $-I$ colors of continuumdominated sources like stars and normal galaxies. Therefore, this analysis should separate any $\mathrm{HH}$ objects from stars and galaxies. First, synthetic colors for stars with $T_{\text {eff }}$ in the range $20,000 \mathrm{~K}$ to $1500 \mathrm{~K}$ are plotted (blue squares). These were calculated for the wavelength ranges of the FORS1 filters using a simple blackbody model. The hottest stars lie close to the origin while the coolest BDs lie in the tail at the end of the sequence (upper right). The direction of the reddening is also plotted. In this figure the colors for both the TW Hya and Cha I star-forming regions (based on the FOV of our images) are also plotted. The idea is to compare the colors of the sources in our images of the 2M1207 region not only to the synthetic colors but also to another star-forming region and any known 

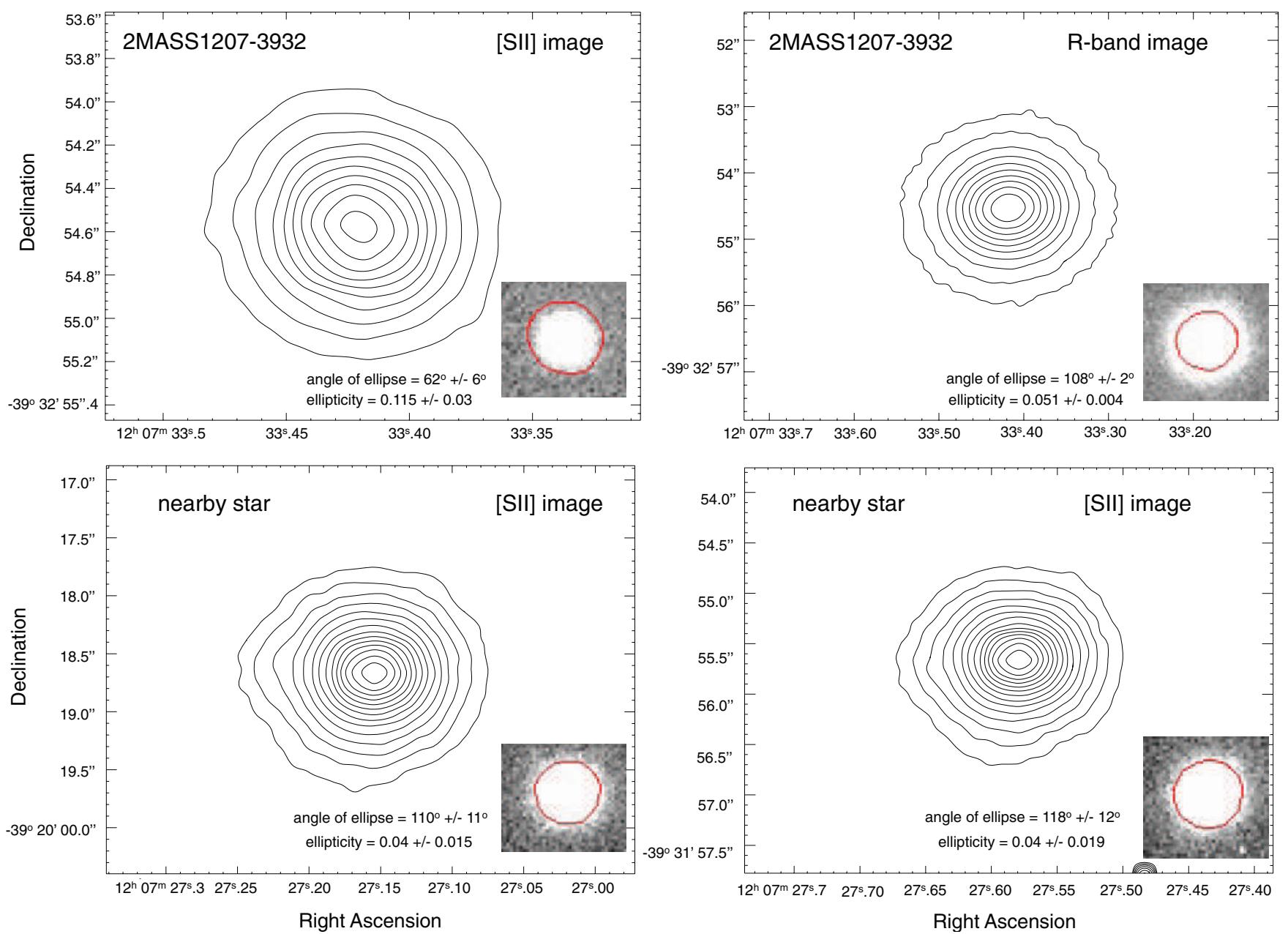

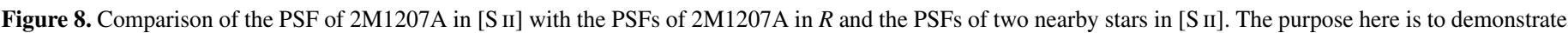

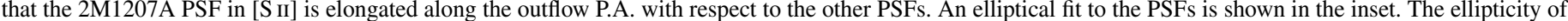

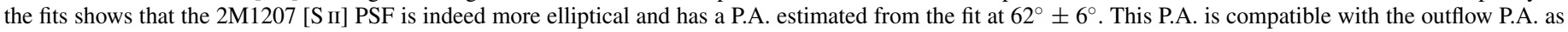
estimated using SA. We use this comparison as strong evidence that we are detecting the 2M1207A outflow in the [S II] image.

(A color version of this figure is available in the online journal.)

$\mathrm{HH}$ objects within that region. Cha I was chosen as suitable images were available in the ESO archive which included the protostar ESO-H $\alpha 574$ and its outflow (Bacciotti et al. 2011). The black triangles represent the sources in the FOV of the TW Hya images and the black asterisks the sources in Cha I. For both star-forming regions, the sources follow the same sequence as the synthetic colors with the hottest objects clustering around the origin and the coolest in the tail.

Looking first at the TW Hya data, the purple triangles are galaxies that could be identified in the field and the green triangles represent features A and D. B and C were omitted as they were too faint for their colors to be measured. While A lies amongst the stars and galaxies ruling out the possibility that it is an HH object, D is offset from the stellar sequence and is bluer than the stars and galaxies plotted. Thus considering the bluer nature of $\mathrm{D}$, that it lies along the outflow P.A., and that it is bow shaped with the bow pointing away from the $\mathrm{BD}$, we conclude that it is an HH object in the 2M1207A outflow. This conclusion is further strengthened by comparison with the knots in the ESO-H $\alpha 574$ outflow. ESO-H $\alpha 574$ powers a well-developed bipolar jet first observed by Comerón \& Reipurth (2006). Comerón \& Reipurth (2006) identified four knots in the blueshifted lobe of the ESO-H $\alpha 574$ flow which they also labeled $\mathrm{A}-\mathrm{D}$ and Knot $\mathrm{E}$ in the redshifted flow. Here we label them HA574-A to HA574-D, to distinguish them from the features in the 2M1207A flow. In the images, HA574-E was the only knot for which the colors could be measured as the others were not well enough separated and too faint in the $I$ band. Its color is represented in Figure 12 by the purple asterisk. HA574-E is also bluer and well separated from the stellar sequence as expected. The fact that it is bluer than $\mathrm{D}$ can be explained by the relatively weak $\left[\mathrm{S}_{\mathrm{II}}\right] \lambda \lambda 6716$, 6731 emission from D compared to HA574-E. Flux-calibrated spectra of the ESO-H $\alpha 574$ outflow which included HA574-A, HA574-B, and HA574-E were also available (J. Alcala, private communication; also see Bacciotti et al. 2011). These spectra were used to estimate the colors for these three knots (purple squares). The color for HA574-E lies close to that estimated from the images while there is a spread in the colors of HA574-A and HA574-B, again consistent with differences in shock conditions.

\section{DISCUSSION AND CONCLUSIONS}

Of the small number of $\mathrm{BD}$ outflows studied to date, $2 \mathrm{M} 1207 \mathrm{~A}$ is perhaps the most interesting due to its low mass, age of some $10 \mathrm{Myr}$, and binarity. With the new results presented here we add considerably to our 2007 paper reporting the 

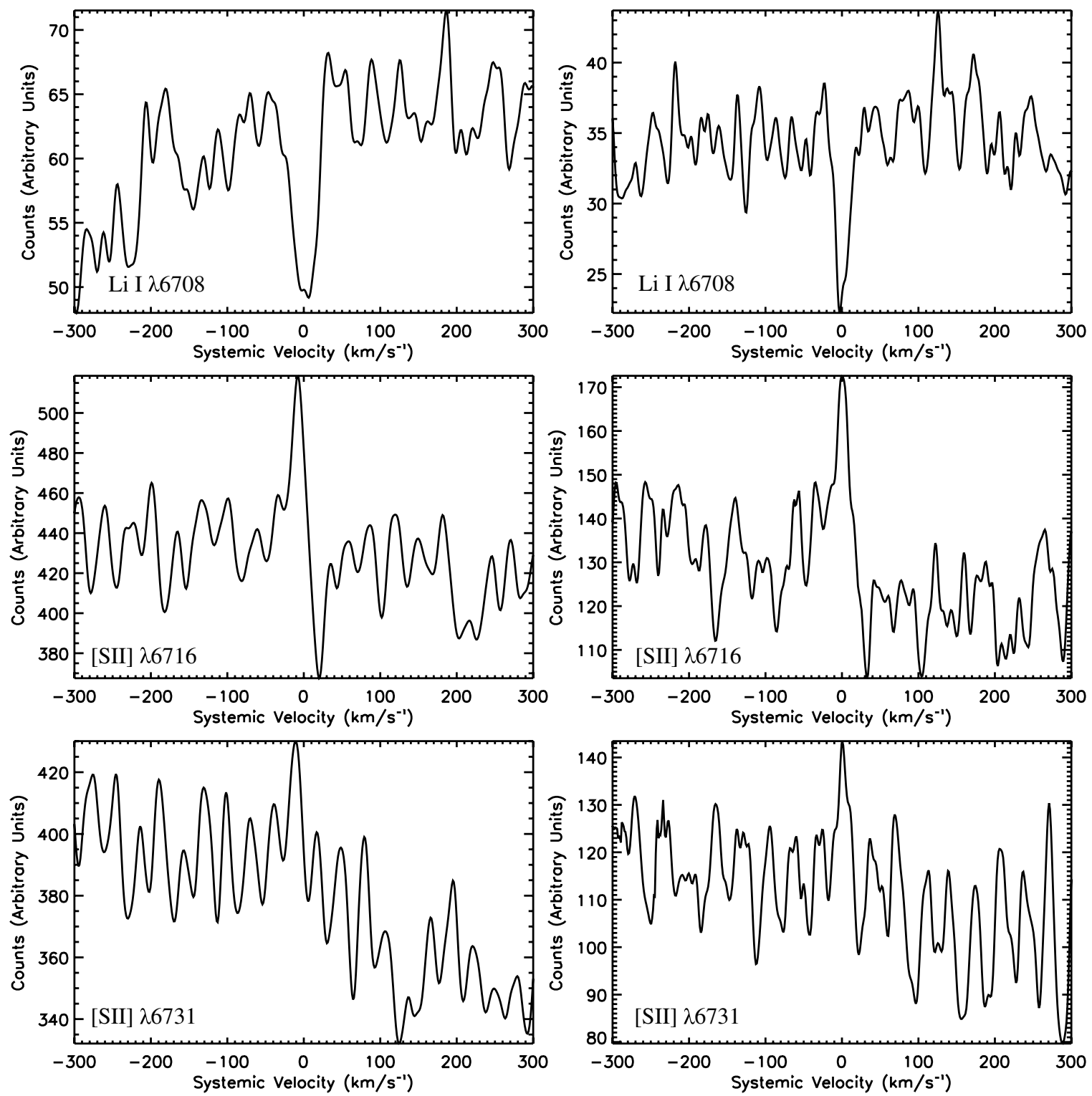

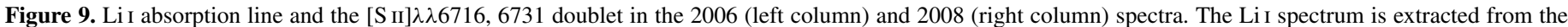

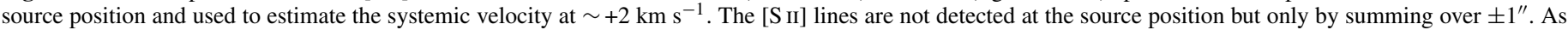
explained in the text this is likely due to the lower critical density of the [S II] lines compared to the [O I] 26300 line.

discovery of this outflow. The 2008 spectra confirm the original results, resolve for the first time for a BD the optical outflow, and allow an estimate of the outflow P.A. to be made. Furthermore, the outflow is detected in the $[\mathrm{S}$ II] image in the form of an elongation in the PSF and at least one HH object (feature D). Evidence in support of the extended features B-D being part of the 2M1207A outflow includes the fact that they lie along the estimated outflow P.A., that the terminal feature D is bow-shock shaped with the apex pointing away from the source, and that $\mathrm{D}$ is separated in Figure 12 from the stars and galaxies. While the color-color analysis shown in Figure 12 demonstrates that at least $\mathrm{D}$ is a probable $\mathrm{HH}$ object, it shows that $\mathrm{A}$ is a star or galaxy. New higher $\mathrm{S} / \mathrm{N}$ images would greatly add to current understanding of this outflow, as first one would be able to study the features with a much improved $\mathrm{S} / \mathrm{N}$ and second a proper motion study, which would confirm the origin of $B$ and $\mathrm{C}$, could be conducted. Using the upper estimate of the disk inclination angle given by Riaz et al. (2012a, 2012b) $\left(69^{\circ}\right)$ and combining it with the measured radial velocities, we estimate the tangential velocity of the 2 M1207A outflow at $\sim 20 \mathrm{~km} \mathrm{~s}^{-1}$. While at the lower end of the range, this is in line with previous estimates of tangential velocities for a sample of BD outflows given by Whelan et al. (2009b). Also the non-detection of a spectro-astrometric signal for $\mathrm{H} \alpha$ is consistent with a low tangential velocity as with such low velocity one would expect only weak shocks and hardly any ionization of $\mathrm{H}$ in the jet and therefore no $\mathrm{H} \alpha$. A velocity of $\sim 20 \mathrm{~km} \mathrm{~s}^{-1}$ would give feature $\mathrm{D}$ an age of some 173 years. Assuming that any new images of 2M1207A would be observed in 2013 January at the earliest, this would mean a $\sim 57$ month time difference between these and the 2008 images. Thus a shift of at least $\sim 0$.'5 in features B-D would be expected, if they are indeed part of the outflow. Such proper motions should be easily measured. The SUSI 2 images shown in Figure 10 were obtained in 2006 January, meaning that a shift of $\sim 0^{\prime \prime} .2$ could reasonably be expected in the knots in the $I$ band. Attempts to measure such a proper motion between these two sets of I-band images were inconclusive. A high-quality spectrum along the P.A. of the outflow to search 

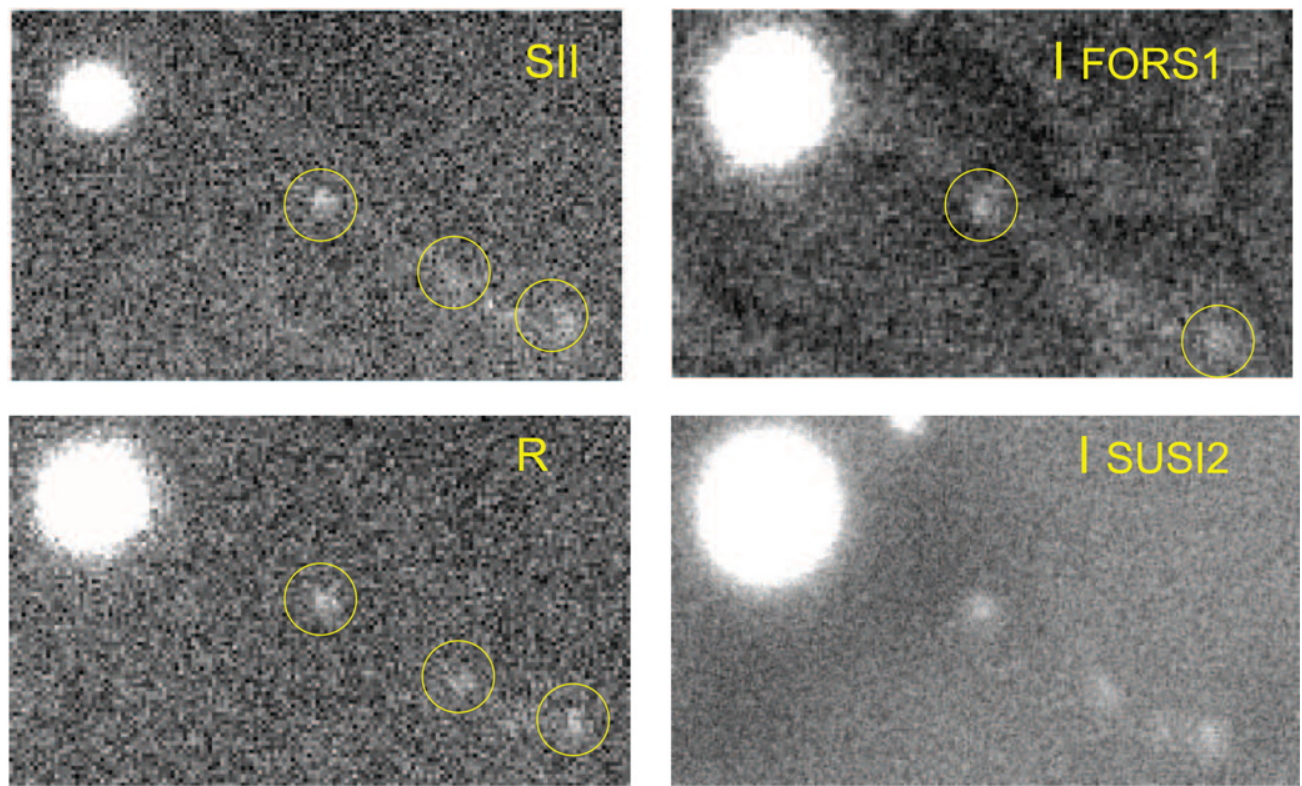

Figure 10. Here the knot-like features seen to the southwest of $2 \mathrm{M} 1207 \mathrm{~A}$ with a P.A. of $\sim 245^{\circ}$ are compared in the $[\mathrm{S}$ II], $R$-band, and $I$-band images. Two of the emission features are detected in the FORS1 I-band image. They are also shown in an SUSI2 image taken two years previous to the FORS1 observations.

(A color version of this figure is available in the online journal.)

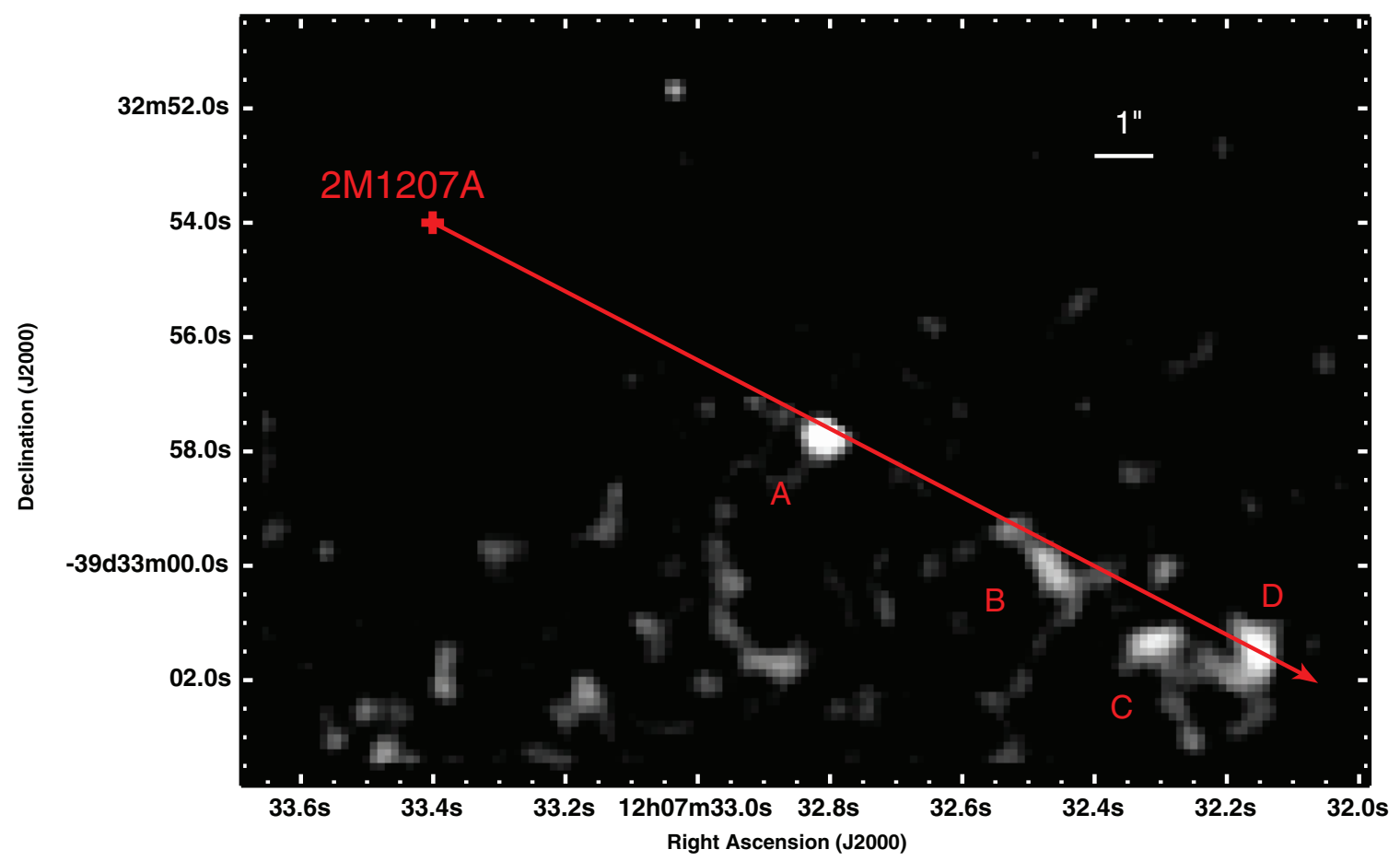

Figure 11. Gaussian-smoothed [S II]-I image. Four separate emission features are revealed with the final one having a clear bow-shock shape. The positions of the features are well fitted with a P.A. of $245^{\circ}$ which is the same P.A. as estimated from the spectro-astrometry.

(A color version of this figure is available in the online journal.)

for shock-related emission lines would also be of great benefit. New imaging and spectroscopic observations would also enable a search for emission features along the blueshifted outflow to be conducted. Finally, we note that while it is normally the case that the blueshifted lobe is preferentially detected, there are cases where the redshifted flow is more prominent (Woitas et al. 2002; Whelan et al. 2009b).

Results presented here also demonstrate that the 2M1207A outflow is well collimated. This confirms that BD outflows can be collimated like low-mass protostellar jets, further adding to the properties they share (Whelan et al. 2009b). As BD optical outflows had not been directly spatially resolved previous to this work, the extent of their collimation was uncertain. However, the detection of a molecular outflow from ISO-Oph 102 by PhanBao et al. (2008) did point to some degree of focussing, as it is a collimated jet which is postulated to be driving the molecular outflows from low-mass stars (Bachiller 1996). Measurements of jet width as a function of distance along the jet for classical 


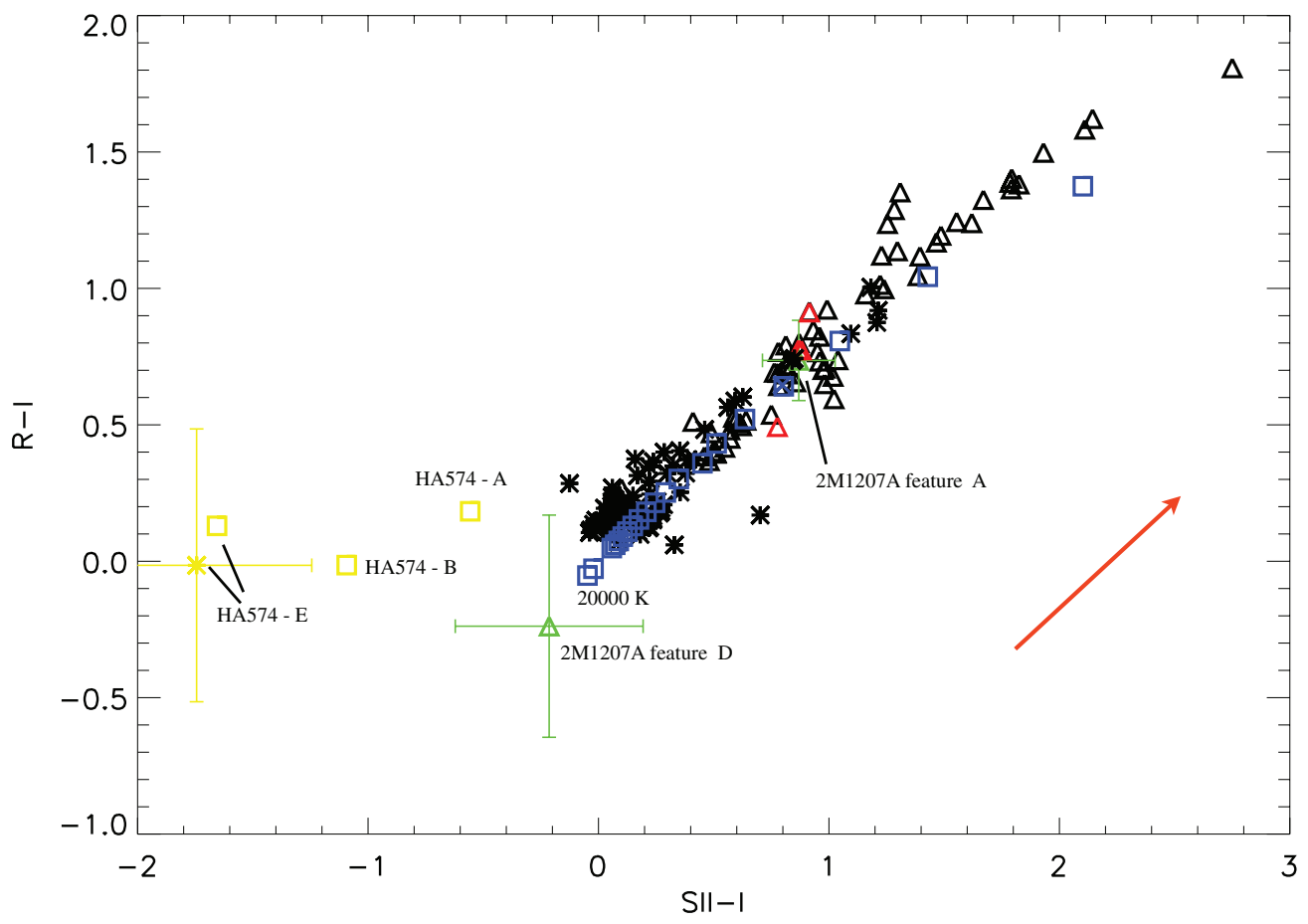

Figure 12. Using a color-color analysis to separate $\mathrm{HH}$ objects from stars and galaxies. The aim of this analysis is to compare the colors of the objects in the TW Hya images with synthetic colors and with an analysis of the protostar ESO-HA 574 and its associated HH objects, in order to test if the faint knot-like features A and D are also HH objects. The squares represent synthetic colors, the circles represent colors estimated from spectra, the triangles represent objects in TW Hya included in the FOV of the 2M1207 images, and the asterisks represent objects in Cha I included in the FOV of the ESO-HA 574 images. The red arrow is the reddening vector. Specifically, the blue squares are synthetic colors for blackbodies in the range 20,000-1500 K, calculated in the range of the FORS1 filters. The purple circles are the color estimates for the knots HA574-A, HA574-B, and HA574-E, calculated from XSHOOTER spectra. The black asterisks are stars in Cha I and the purple asterisk marks the color of HA574-E measured from the image. The black triangles are stars in the TW Hya region, the purple triangles are identified galaxies, and the green triangles features A and D. The marked errors in the colors for HA574-E and A and D are quite large while the errors for the other points are found to be $<0.02$. The position of feature D, the bow-shock-shaped feature, lies with the other known $\mathrm{HH}$ objects while A lies amongst the stars and galaxies. Based on this analysis and other evidence discussed, we conclude that D is a shock in the 2M1207A outflow.

T Tauri stars (CTTSs) have shown that collimation occurs on scales of a few tens of an AU from the star (Ray et al. 2007) which is consistent with magnetohydrodynamical models. As well as being collimated, jets from low-mass stars are also often episodic in that they are made up of a series of distinct knots assumed to be associated with separate accretion events. When discussing episodic outflows the striking $\mathrm{H}_{2}$ flow known as $\mathrm{HH}$ 212 , driven by a Class 0 young low-mass star, immediately comes to mind (Zinnecker et al. 1998). The HH 212 flow is very well defined with matching pairs of bow-shock-shaped knots on either side of the driving source. By studying the individual knots in protostellar jets it is possible to get a picture of the mass loss and accretion history of the source. Our study strongly indicates that accretion and outflow activity proceeds in this extremely low-mass object in an analogous way to low-mass protostars, and by studying this flow we will be able to investigate the history of the 2M1207 system.

As the current sample of known BD outflows is small, the present aim of our study of BD outflow activity is to greatly increase the sample. The approach of using SA to identify BD outflows, while effective, is slow as high-quality, high $\mathrm{S} / \mathrm{N}$ spectra are required and thus observations with an $8 \mathrm{~m}$ class telescope or better are generally necessary. In addition, if one wants to thoroughly investigate the properties of the outflow, including the morphology, kinematics, and mass-loss rate, spectra along the outflow P.A. are needed. Hence, to identify the outflow sources and constrain the outflow P.A., two spectra at orthogonal P.A.s should be observed and then a follow-up spectrum must be taken along the estimated P.A. We consider that the imaging observations of the 2M1207A outflow presented here offer an underutilized method of identifying BD outflows. An [S II] imaging study to look for extensions in the PSFs of BD candidates would be a fast way of identifying outflows as more than one source could be observed at the same time and we would expect to reach a better $\mathrm{S} / \mathrm{N}$ in any [S II] outflow emission with an imager than a spectrograph for comparable exposure times. A serious limitation to imaging studies of jets from low-mass protostars is the strength of the continuum emission which is many times stronger than the jet emission. For this reason the jet is often not seen in the image until several arcseconds from the source. As the continuum emission in the vicinity of the $[\mathrm{S}$ II $] \lambda \lambda 6716$, 6731 lines in BDs and VLMSs is fainter than in CTTSs, this enhances the possibility of detecting any outflows in an imaging study.

Furthermore, a significant number of the BDs/VLMSs shown spectroscopically to have outflows (four out of six) also have near-edge-on disks (Whelan et al. 2009a, 2009b; Bacciotti et al. 2011). The effect of this is to obscure the emission from the BD or star and further enhance the contrast between the jet and continuum emission in favor of the jet emission. For example, the $100 M_{\mathrm{JUP}}$ mass object Par-Lup3-4 is known to be underluminous and an examination of archived FORS1 images and UVES spectra allows the ratio of the $\left[\mathrm{S} \mathrm{II}_{\mathrm{II}}\right.$ jet emission to continuum emission in the range of the $\left[\mathrm{S}_{\mathrm{II}}\right]+62$ filter to be estimated at $50 \%$. Thus, the Par-Lup3-4 jet is easily detected in the FORS image (Fernández \& Comerón 2005). We are confident of repeating the imaging observations of 2M1207A 
discussed here for other BDs and especially those with edge-on disks. Such observations would of course immediately give the jet P.A. allowing for a more detailed follow-up study of accretion and outflow activity from high-quality spectra.

E. T. Whelan is supported by an IRCSET-Marie Curie International Mobility Fellowship in Science, Engineering, and Technology within the 7th European Community Framework Programme; T. P. Ray acknowledges support from Science Foundation Ireland under the Research Frontiers Program grant 11/RFP/AST/3331; P. J.Kavanagh is funded through the BMBF/DLR grant 50-0R-1009. E. T. Whelan also acknowledges support from the European Southern Observatory under the visitor programme.

\section{REFERENCES}

Appenzeller, I., Bender, R., Böhnhardt, H., et al. 2000, in From Extrasolar Planets to Cosmology: The VLT Opening Symposium, ed. J. Bergeron \& A. Renzini (Berlin: Springer), 3

Bacciotti, F., Whelan, E. T., Alcalá, J. M., et al. 2011, ApJ, 737, L26

Bachiller, R. 1996, ARA\&A, 34, 111

Barrado y Navascués, D. 2006, A\&A, 459, 511

Brugel, E. W., Boehm, K. H., \& Mannery, E. 1981, ApJS, 47, 117

Chauvin, G., Lagrange, A.-M., Dumas, C., et al. 2004, A\&A, 425, L29

Comerón, F., \& Fernández, M. 2011, A\&A, 528, A99

Comerón, F., Fernández, M., Baraffe, I., Neuhäuser, R., \& Kaas, A. A 2003, A\&A, 406, 1001

Comerón, F., \& Reipurth, B. 2006, A\&A, 458, L21

Dekker, H., D’Odorico, S., Kaufer, A., Delabre, B., \& Kotzlowski, H. 2000, Proc. SPIE, 4008, 534

Fernández, M., \& Comerón, F. 2001, A\&A, 380, 264

Fernández, M., \& Comerón, F. 2005, A\&A, 440, 1119
Ferreira, J., Dougados, C., \& Cabrit, S. 2006, A\&A, 453, 785

Hartigan, P., Edwards, S., \& Ghandour, L. 1995, ApJ, 452, 736

Machida, M. N., Inutsuka, S.-i., \& Matsumoto, T. 2009, ApJ, 699, L157

Mohanty, S., Jayawardhana, R., \& Barrado y Navascués, D. 2003, ApJ, 593, L109

Mohanty, S., Jayawardhana, R., \& Basri, G. 2005, ApJ, 626, 498

Mohanty, S., Jayawardhana, R., Huélamo, N., \& Mamajek, E. 2007, ApJ, 657,1064

Mundt, R., Stocke, J., \& Stockman, H. S. 1983, ApJ, 265, L71

Osterbrock, D. E. 1988, PASP, 100, 412

Phan-Bao, N., Riaz, B., Lee, C.-F., et al. 2008, ApJ, 689, L141

Podio, L., Garcia, P. J. V., Bacciotti, F., et al. 2008, A\&A, 480, 421

Ray, T., Dougados, C., Bacciotti, F., Eislöffel, J., \& Chrysostomou, A. 2007, in Protostars and Planets V, ed. B. Reipurth, D. Jewitt, \& K. Keil (Tucson, AZ: Univ. Arizona Press), 231

Riaz, B., \& Gizis, J. E. 2008, ApJ, 681, 1584

Riaz, B., Lodato, G., Stamatellos, D., \& Gizis, J. E. 2012a, MNRAS, 424, L74

Riaz, B., Lodato, G., Stamatellos, D., \& Gizis, J. E. 2012b, MNRAS, 422, L6

Scholz, A., Jayawardhana, R., \& Brandeker, A. 2005, ApJ, 629, L41

Stelzer, B., Scholz, A., \& Jayawardhana, R. 2007, ApJ, 671, 842

Szeifert, T., Appenzeller, I., Fuertig, W., et al. 1998, Proc. SPIE, 3355, 20

Takami, M., Bailey, J., Gledhill, T. M., Chrysostomou, A., \& Hough, J. H. 2001, MNRAS, 323, 177

Wang, H., \& Henning, T. 2006, ApJ, 643, 985

Whelan, E., \& Garcia, P. 2008, in Jets from Young Stars II, ed. F. Bacciotti, L. Testi, \& E. Whelan (Lecture Notes in Physics, Vol. 742; Berlin: Springer), 123

Whelan, E. T., Dougados, C., Perrin, M. D., et al. 2010, ApJ, 720, L119

Whelan, E. T., Ray, T. P., \& Bacciotti, F. 2009a, ApJ, 691, L106

Whelan, E. T., Ray, T. P., Bacciotti, F., et al. 2005, Nature, 435, 652

Whelan, E. T., Ray, T. P., \& Davis, C. J. 2004, A\&A, 417, 247

Whelan, E. T., Ray, T. P., Podio, L., Bacciotti, F., \& Randich, S. 2009b, ApJ, 706, 1054

Whelan, E. T., Ray, T. P., Randich, S., et al. 2007, ApJ, 659, L45

Woitas, J., Ray, T. P., Bacciotti, F., Davis, C. J., \& Eislöffel, J. 2002, ApJ, 580,336

Zinnecker, H., McCaughrean, M. J., \& Rayner, J. T. 1998, Nature, 394, 862 